\title{
Liquid-Phase Polymer-Based Retention: Theory, Modeling, and Application for the Removal of Pollutant Inorganic Ions
}

\author{
Manuel Palencia \\ Department of Chemistry, Faculty of Natural and Exact Science, University of Valle, Cali, Colombia \\ Correspondence should be addressed to Manuel Palencia; manuel.palencia@correounivalle.edu.co
}

Received 10 December 2014; Accepted 9 February 2015

Academic Editor: Marinos Pitsikalis

Copyright ( 2015 Manuel Palencia. This is an open access article distributed under the Creative Commons Attribution License, which permits unrestricted use, distribution, and reproduction in any medium, provided the original work is properly cited.

\begin{abstract}
The aim of this work is to give the theoretical background of liquid-phase polymer-based retention (LPR) technique, provide a complete model of separation process, and evaluate its application for the removal of pollutant inorganic ions (PIIs) from aqueous solution. Thus, fundaments of LPR modeling by washing method from the ion distribution in active and passive zones are specified and its application was evaluated for cadmium removal using poly(sodium styrene sulfonate) as water-soluble polymer (WSP). LPR simulations were performed using a semiempirical description from data of concentration, filtration factorm, and retention coefficients. It is concluded that semiempirical simulation of PII retention by WSP can be performed from a small amount of experimental data using the described modeling. In addition, new approximations associated with molecular structure of polymer should be performed and linked to parameters of LPR experiments.
\end{abstract}

\section{Introduction}

The aim of this work is to give the theoretical background of LPR technique, provide a complete model of separation process, and evaluate its application for the removal of pollutant inorganic ions (PIIs) from aqueous solution. Thus, to start, it is important to indicate that membrane separation processes are very attractive for waste, pollutant natural and drinking water treatment due to their simplicity of operation and moderate energy consumption $[1,2]$. In the case of ultrafiltration (UF), this technique has been applied to the treatment of effluents that contain particulate materials, dissolved species with high molecular weight, and metal ions when a strength membrane-metal interaction is present [1-5]. In UF systems, the membrane acts as a porous barrier at molecular level, but the pore size is too large that it is not possible to retain dissolved inorganic ions unless these are present in a colloidal form. On the other hand, the addition of water-soluble high molecular weight species to the UF unit with a strength polymer-ion interaction leads to ion retention by the formation of new macromolecular species which can be easily retained by sizeexclusion mechanism. Moreover, metal selective extraction can be achieved if specific chelating or ionic groups are bonded on the polymeric chains $[3,5]$. This technique has been named in different ways: liquid-phase polymer-based retention (LPR) $[3,5,6]$, polymer assisted ultrafiltration (PAUF) [7, 8], ultrafiltration-complexation [9-13], polymer or polyelectrolyte enhanced ultrafiltration (PE-UF) [14-18], or, simply, enhanced ultrafiltration $[19,20]$.

The principle of LPR was firstly suggested by Michaels in 1968 and Blatt et al. (1968) presented a theoretical discussion to connect the method of analysis by diafiltration with continuous ultrafiltration [20]. Geckeler et al. (1986) carried out the first experimental advances and analytical applications related to this technique $[6,21,22]$. Later, many research groups have worked on the evaluation and description of retention properties of different water-soluble polymers (WSPs) for environmental and analytical applications [5, 2130]; it is important to highlight the works done by Rivas et al. related to the modeling and development of new experimental configurations and novel applications of this technique $[3,31,32]$.

\section{LPR: Theory and Modeling}

LPR technique is a hybrid membrane separation method which combines UF membranes with WSPs in order to 


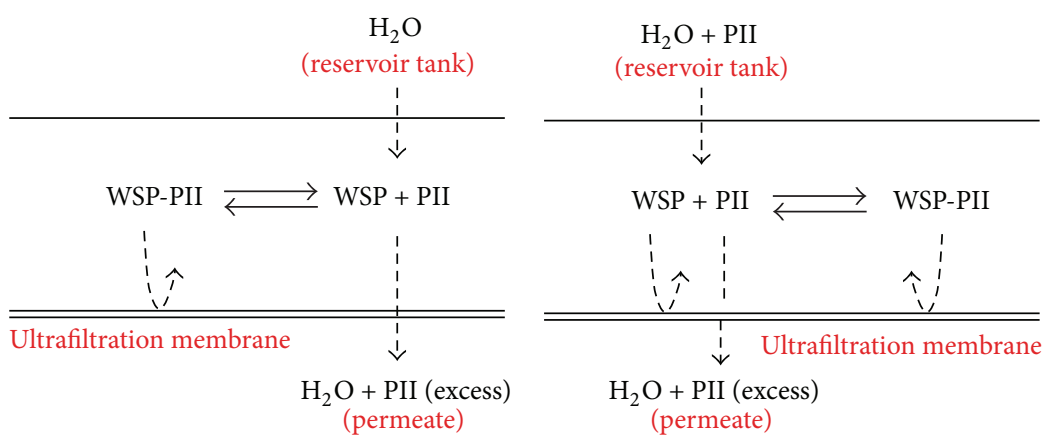

(a)

(b)

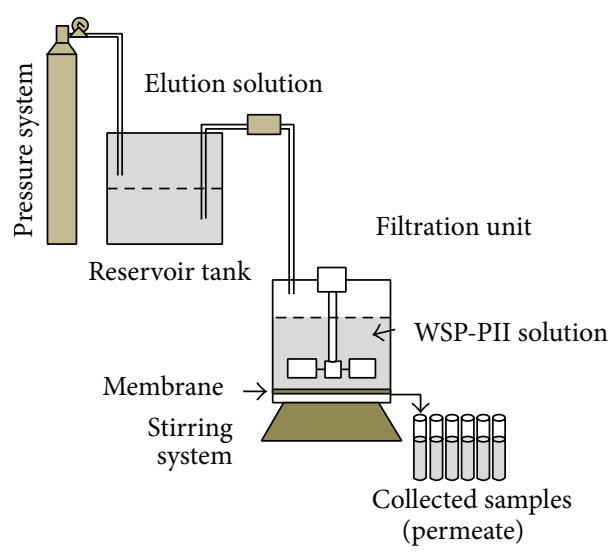

(c)

FIGURE 1: Description of LPR technique by washing method (a) and enrichment method (b) and schematic representation of experimental device (c).

separate low molecular weight species dissolved in aqueous solution [5]. Thus, a WSP solution and a metal ion solution are contacted in the feed-side of an UF system; the metal ions are consequently bonded to the polymer and new macromolecular species are formed and retained by a sizeexclusion mechanism while the unbounded species, with a diameter smaller than cutoff diameter of the membrane, passes to the permeate stream $[5,20]$.

Usually LPR technique can be applied by two operation methods: (1) the washing method or elution method (with the ionic strength kept constant or not) which is the most used method to study the PII's retention capacity of a polymer in aqueous solution and is a discontinuous separation process and (2) the enrichment method or concentration method which is a continuous separation process [5]. In the washing method, a certain amount of polymer and PII solutions with known concentrations are placed in the feed-side of an UF cell and a water stream is passed through the cell $[5,22,25]$. On the other hand, for the application of the enrichment method, the WSP solution is placed in a stirred cell and a PII solution is added continuously from a pressurized reservoir. However, when the bonding groups of the WSP are saturated by their interaction with the PII in the solution, the maximum retention capacity (MRC) of polymer is reached and the process cannot continue $[5,20]$. Evidently, the physicochemical properties of the WSP and the polymer-metal complexes must be taken into account and their interaction with the membrane surface is also very relevant. During the retention and separation process by LPR, the permeate is removed at the same rate, keeping the volume in the feed cell and the polymer concentration constant all the time [20]. Reviews on LPR technique have been performed by Geckeler et al. $[6,21,22]$ and most recently by Rivas et al. $[3,5]$ (see Figure 1).

The LPR technique can be visualized like cyclic separation process and thus it should be economically more feasible when WSP can be regenerated and reused. Among regeneration methods of the WSP are the chemical methods (protolysis, transcomplexation, and redox reaction), electrochemical methods, and thermal methods [3]. In this way, new options of regeneration of chelating groups by using new components incorporated to the system can be useful to make the process more feasible.

The LPR technique, either by washing method or by enrichment method, is based on the interaction between the WSP and the PIIs in aqueous solution and the progressive retention by an UF membrane of the new species formed [20]. The type of interaction depends on the chemical nature of the WSP functional groups. In any case, these interactions are mainly due to electrostatic forces and the formation of coordinating bonds. The variables that affect the WSPPII interaction are classified into two groups: intrinsic to the polymer (i.e., the nature of the atoms in the backbone 
chain, the nature of the functional groups attached to the backbone, the structure and composition of the polymer, the molecular weight and the polydispersity, the distance between the functional groups and the backbone, the degree of branching, etc.) and extrinsic to the polymer (i.e., charge and type of the metal ion, the $\mathrm{pH}$ of the solution, the ionic strength, the temperature, and the dielectric constant of the medium) $[3,5,6,12]$.

From a molecular point of view, PII retention by WSP during the application of LPR technique can be understood from the description of three types of main interactions: (1) WSP-PII interaction, (2) membrane-PII interaction, and (3) WSP-membrane interaction [20]. Commonly, WSP-PII interaction has been the attention focus in many researches where it is assumed that retention capacity of polymer is very high in comparison with retention capacity of the membrane and, in consequence, membrane is assumed to be inert in many cases.

If WSP and PII solutions are contacted, the ion distribution in the UF cell is governed by the WSP-PII interactions and the electrostatic attraction between WSP chains and PII in solutions can be discussed by counterion condensation theory (CCT) [33, 34]. There are several approaches to describe counterion condensation in WSP solutions by cell models. In the first approach, two-state model or two-zone model proposed by Oosawa and Manning, the counterions are separated into "free" and "condensed." Free counterions can explore the solution volume (external zone), and the condensed counterions are located within a small volume surrounding the polymer backbone which is assumed to be rodlike (polymer domain or internal zone) [35, 36]. Thus, during LPR technique by washing method, PIIs are distributed in the external and internal zones of a WSP solution and only PIIs distributed in the external zone can be eluted from UF cell to permeate stream. On the other hand, during LPR technique by enrichment method, PIIs are added to the external zone and transported to internal zone as PII concentration in the external zone is continuously increased. In this case, concentration of PIIs in the permeate depends on their distribution between the external and internal zones [31].

As modeling strategy, LPR system can be described as a group of four independent regions contacted with a fifth "passive" region [31]. The regions considered are (1) reservoir (which is a passive component in the washing method, but in other operation modes, such as the enrichment method, it should be considered), (2) solution bulk (which is the fraction of the solution where metal ions are not affected by the interaction with some retainer component of the cell which can be, for example, WSP or membrane), (3) permeate (this is the fraction of solution which can pass through the membrane and corresponds only to solvent and solutes distributed in the solution bulk), (4) membrane surface (which is based in the adsorption process on polymeric surfaces and retention properties of polymer in solid phase when an aqueous stream is passed through it), and (5) polymer domain (which is the region where the strongly interacting counterions with the polymer are distributed) [31]. If the membrane is considered to be an inert component and the polymer is not present in the cell (blank experiment) then regions 1, 2, 3, and 4 can be considered under a hydrodynamic control and system can be described from filtration theories; when the polymer is present and membrane is not inert, regions 2,4 , and 5 are assumed to be controlled by two factors: a hydrodynamic control resulting from its interaction with regions 1,3 , and 4 and a physicochemical control related to the WSP-PII and membrane-PII interactions.

Under the consideration of ion distribution interacting regions, the filtration cell is like a continuously stirred tank reactor, and therefore it is identical in design requirements to the batch reactor except for the introduction of a constant flow of feed and a constant removal of reactor contents so that the reactant volume in the reactor remains constant. Thus, the filtration cell is inherently a steady state reactor. This is accomplished by keeping the volume, temperature, feed rate, and pressure of the filtration cell constant during the experiment.

To describe the LPR technique by "ion distribution model" for washing method, system retention $\left(R_{\text {syst }}\right)$ is the result of PII transport between regions $2 \rightarrow 4$ (from bulk of solution to membrane surface) and between regions $2 \rightarrow 5$ (from bulk of solution to polymer domain) at $t=0$. In a long enough time (if $t \rightarrow \infty$ then $F \rightarrow \infty ; F$ is defined to be the "filtration factor" and is equal to the volume of permeate, $V_{p}$, divided by solution volume in the cell, $V_{c}$ ) a new distribution of PII in the system is obtained. In this case, retention by the polymer is the fraction of PII distributed in the polymer domain $\left(R_{\mathrm{pol}}\right)$ and retention by the membrane is the fraction of PIIs distributed in the surface polymer $\left(R_{m}\right)$ which cannot be eluted by continuous addition of solvent from reservoir in the experimental conditions of pressure, temperature, $\mathrm{pH}$, ionic strength, polymer-metal ratio, and so forth. Thus, different situations can be analyzed: (1) modeling of inert system, (2) membrane-PII interaction parameter (modeling of blank experiment), (3) WSP-PII interaction parameter (modeling of LPR in presence of WSP considering the membrane as an inert component), and (4) WSP-membrane-PII interaction parameters [31].

For the first case, modeling of inert system, if an inert system is considered then PIIs can be distributed in regions 2 and 3. In this case, from the following mass balance

$$
N_{c}=N_{0}-N_{p} \text {, }
$$

where $N_{c}$ and $N_{p}$ are the number of moles distributed in regions 2 and 3 , respectively, and $N_{0}$ is the number of moles initially placed in the cell. Since $d N_{c}=V_{c} d C_{c}$ and $d N_{p}=$ $d\left(V_{p} C_{p}\right)=V_{p} d C_{p}+C_{p} d V_{p}$, it is obtained that

$$
-V_{c} d C_{c}=V_{p} d C_{p}+C_{p} d V_{p}
$$

where $C_{c}$ and $C_{p}$ are cell and permeate concentrations, respectively. Since the mathematical expression described by (2) relates three interdependent variables, $d C_{c}, d C_{p}$, and $d V_{p}$, the assumption of a linear approximation is useful in order to obtain an analytical resolving of (2). Thus, for small values of $\Delta F$ (where $\Delta F=F_{n}-F_{n-1}$ for the $n$th fraction recollected of permeate) it can be assumed that

$$
d C_{c}=\alpha d C_{p}
$$


$\alpha$ being an empirical parameter which describes the relative changes of $C_{c}$ and $C_{p}$ as functions of $F$. By considering of a linear dependence $C_{c}$ and $C_{p}$, from (2) and (3),

$$
\frac{d C_{p}}{d V_{p}}=\frac{-C_{p}}{\alpha V_{c}+V_{p}} .
$$

Resolving and evaluating for boundary conditions at $t=0$ and $t_{1}$ (at $t=0, C_{p}=C_{0}$ and $V_{p}=0$; at $t_{1}, C_{p}=C_{p}$ and $\left.V_{p}=V_{p}\right)$ are obtained:

$$
\frac{C_{p}}{C_{0}}=\frac{\alpha V_{c}}{\alpha V_{c}+V_{p}}=\frac{\alpha}{\alpha+F},
$$

where $C_{p}$ and $V_{p}$, in terms of LPR experiments, are given by

$$
\begin{gathered}
C_{p}=\frac{1}{V_{p}} \sum_{i=1}^{n}\left(C_{p, i} V_{i}\right), \\
V_{p}=\sum_{i=1}^{n} V_{i},
\end{gathered}
$$

where $V_{i}$ and $C_{p, i}$ are the volume and the concentration of each one of the permeate fractions recollected.

Note that $\alpha$ can be easily calculated from experimental measures. Thus, one value of $\alpha$ can be obtained from a plot of $C_{c}$ versus $C_{p}$. For the situation where $C_{c}$ is not linear with respect to $C_{p}$, several values of $\alpha$ can be assigned depending on the selection of approximately linear ranges defined according to the experimental data.

For the second case, membrane-metal interaction parameter (modeling of blank experiment), for blank experiment, PIIs can be distributed in regions 2, 3, and 4 since membrane is not assumed to be an inert component of the system. Describing the PII adsorption on the membrane surface by means of Donnan's equilibrium given by

$$
\mathrm{M}^{\mathrm{n}+}(\mathrm{sol}) \rightleftarrows \mathrm{M}^{\mathrm{n}+} \text { (membrane) }
$$

the following membrane-PII interaction constant $\left(k_{m}\right)$ can be defined:

$$
k_{m}=\frac{C_{m}}{C_{c}}=\frac{N_{m} V_{c}}{N_{c} w_{m}},
$$

where $C_{m}$ is the number of PII moles adsorbed $\left(N_{m}\right)$ per unit of membrane mass $\left(W_{m}\right)$. Thus, $k_{m}$ is defined to be equal to distribution coefficient analogously to the strategy used to describe the affinity between PII and polymeric resin [16].

From (8) it is concluded that $d N_{m}=k_{m} w_{m} d C_{c}$, where $N_{m}$ is the number of moles in region 4 . Thus, from mass balance in regions 2, 3, and 4 the following relation is obtained:

$$
-V_{c} d C_{c}=V_{p} d C_{p}+C_{p} d V_{p}+k_{m} w_{m} d C_{c} .
$$

By (3) and (9)

$$
\frac{d C_{p}}{d V_{p}}=\frac{-C_{p}}{\left(V_{c}+k_{m} w_{m}\right) \alpha+V_{p}}
$$

Note that, implicitly, it was assumed that retention properties of membrane are not affected by the elution of PIIs in the other regions. This assumption is strongly dependent on nature of membrane-PII interaction. However, for high levels of membrane-PII interaction can be appropriate since the adsorption on membrane surface will be favored, and for low levels of membrane-PII interaction, the membrane can be considered an inert component. In consequence, the model considers that the initial concentration of PII in the cell at $t=0$ is modified by membrane-PII interaction and therefore an effective initial concentration $C_{0}$ can be obtained.

Resolving and evaluating for boundary conditions at $t=0$ and $t_{1}$

$$
\frac{C_{p}}{C_{0}}=\frac{\left(1+k_{m} w_{m} V_{c}^{-1}\right) \alpha}{\left(1+k_{m} w_{m} V_{c}^{-1}\right) \alpha+F}=\frac{\alpha \theta_{m}}{\alpha \theta_{m}+F},
$$

where $\theta_{m}$ can be defined as a system interaction parameter given by $\theta_{m}=1+k_{m} w_{m} V_{c}^{-1}$.

For the third case, WSP-PII interaction parameter (modeling of LPR in presence of WSP considering the membrane as an inert component), it is seen that it corresponds to one typical assumption done for LPR in retention experiments. This situation is achieved as a result of adequate selection of the material of the membrane or by control of the experimental conditions (e.g., $\mathrm{pH}$ and ionic strength). According to the proposed model, PIIs can be distributed in regions 2, 3, and 5 , and the polymer WSP-PII interaction constant $\left(k_{1}\right)$ can be described through Donnan's equilibrium between polymer domain and bulk of solution according to Manning's theory which is given by

$$
\mathrm{M}^{\mathrm{n}+}(\text { sol }) \rightleftarrows \mathrm{M}^{\mathrm{n}+} \text { (polymer) } .
$$

Thus, $k_{\text {pol }}$ can be defined as

$$
k_{1}=\frac{C_{\mathrm{pol}}}{C_{c}}=\frac{N_{b}}{C_{c} V_{\mathrm{pol}}} .
$$

From (13) it is concluded that $d N_{b}=k_{\text {pol }} V_{\text {pol }} d C_{c}$, where $N_{b}$ is the number of moles in region 5 and $V_{\text {pol }}$ is the volume of polymer domain. From mass balance in regions 2,3 , and 5 the following relation is obtained:

$$
-V_{c} d C_{c}=V_{p} d C_{p}+C_{p} d V_{p}+k_{\mathrm{pol}} V_{\mathrm{pol}} d C_{c}
$$

By (3) and (14),

$$
\frac{d C_{p}}{d V_{p}}=\frac{-C_{p}}{\left(V_{c}+k_{\mathrm{pol}} V_{\mathrm{pol}}\right) \alpha+V_{p}} .
$$

Solution of (15) is analogous to (10) and therefore

$$
\frac{C_{p}}{C_{0}}=\frac{\left(1+k_{\mathrm{pol}} V_{\mathrm{pol}} V_{c}^{-1}\right) \alpha}{\left(1+k_{\mathrm{pol}} V_{\mathrm{pol}} V_{c}^{-1}\right) \alpha+F}=\frac{\alpha \theta_{\mathrm{pol}}}{\alpha \theta_{\mathrm{pol}}+F},
$$


TABLE 1: Values of $R_{\text {pol }}$ and $C_{\text {in }}$ for removal of PIIs (PSSNa-Cd) [20, 23, 27, 36].

\begin{tabular}{llllllll}
\hline$C_{\text {in }}(\mathrm{mmol} / \mathrm{L})$ & 0.18 & 0.36 & 0.89 & 1.34 & 2.23 & 3.56 & 4.45 \\
\hline$R_{\mathrm{pol}}(\%)$ & 61.0 & 56.7 & 47.0 & 41.2 & 35.1 & 30.9 & 28.0 \\
\hline
\end{tabular}

where $\theta_{p}$ can be defined as a system interaction parameter given by $\theta_{\mathrm{pol}}=1+k_{\mathrm{pol}} V_{\mathrm{pol}} V_{c}^{-1}$.

Finally, for WSP-membrane-PII interaction parameters, PIIs can be distributed in regions $2,3,4$, and 5 , and $k_{1}$ and $k_{m}$ can be described through Donnan's equilibriums given by (7) and (12) assuming that these interactions can be considered to be independent; that is to say, retention properties related to one specific interaction are not affected by the occurrence of the other. Thus, the change of number of moles distributed in regions 4 and 5 is given, respectively, by $d N_{m}=k_{m} w_{m} d C_{c}$ and $d N_{b}=k_{\text {pol }} V_{\text {pol }} d C_{c}$; therefore

$$
-V_{c} d C_{c}=V_{p} d C_{p}+C_{p} d V_{p}+k_{m} w_{m} d C_{c}+k_{\mathrm{pol}} V_{\mathrm{pol}} d C_{c}
$$

And it can be obtained that

$$
\frac{d C_{p}}{d V_{p}}=\frac{-C_{p}}{\left(1+k_{m} w_{m}+k_{\mathrm{pol}} V_{\mathrm{pol}}\right) \alpha V_{c}+V_{p}}
$$

Solution of (18) is analogous to (10) and (15), and therefore

$$
\frac{C_{p}}{C_{0}}=\frac{\left(1+k_{m} w_{m} V_{c}^{-1}+k_{\mathrm{pol}} V_{\mathrm{pol}} V_{c}^{-1}\right) \alpha}{\left(1+k_{m} w_{m} V_{c}^{-1}+k_{\mathrm{pol}} V_{\mathrm{pol}} V_{c}^{-1}\right) \alpha+F}=\frac{\alpha \theta}{\alpha \theta+F},
$$

where $\theta=1+k_{m} w_{m} V_{c}^{-1}+k_{\mathrm{pol}} V_{\mathrm{pol}} V_{c}^{-1}$.

\section{Materials and Methods}

3.1. Reagents and Filtration Unit. In order to evaluate the LPR modeling, commercially available poly(sodium styrene sulfonate) (PSSNa, Aldrich) was used as WSP. Cadmium nitrate (analytical grade from Merck) was used to prepare the PII solutions. WSP and PIIs were selected because sufficient theoretical and experimental data related to LPR technique are available from previous publications. Polyethersulfone (PESm) disk-shaped membranes (Biomax PBGC; nominal molar mass cutoff of $10 \mathrm{kDa}$, Amicon BioseparationsMillipore Co.) were used in all experiments. Experiments were performed in a stirred-cell filtration unit (Millipore, model 8050). The components and operation modes of the filtration system have been described in previous publications [20] and include a reservoir tank, filtration cell, magnetic stirring system, ultrafiltration membrane, and pressure system (Figure 1(c)).

\subsection{Experimental Procedure}

3.2.1. Retention of PIIs by the Washing Method. In each experiment, $10 \mathrm{~mL}$ of a $20 \mathrm{mmol} / \mathrm{L}$ WSP solution was mixed with $10 \mathrm{~mL}$ of a $3.74 \mathrm{mmol} / \mathrm{L}$ PIIs solution. The working $\mathrm{pH}(2.0,3.5$, and 5.0), operating pressure, and stirring rate were $6.0,300 \mathrm{kPa}$, and $200 \mathrm{rpm}$, respectively. These operating conditions have been previously established [19, 20, 31]. Permeate fractions with a volume of $20 \mathrm{~mL}$ were collected, and the PII concentration was measured by atomic absorption spectroscopy (AAS, Unicam Solaar M5). A blank experiment was performed using the same experimental conditions.

3.2.2. Semiempirical Simulation of LPR Experiments. Experiments were simulated from the experimental data obtained in Section 3.2.1 and by using (19). By combining $R_{\text {syst }}$ for the washing method $\left(R_{\text {syst }}=1-C_{p} F C_{\text {in }}^{-1}\right)$ and (19), $R_{\text {syst }}$ can be expressed as a function of the model parameters

$$
R_{\text {syst }}=1-\frac{\alpha \theta C_{0}}{(\alpha \theta+F) C_{\text {in }}} F .
$$

Consequently, the retention for infinite dilution $\left(R_{\infty}\right.$ for $F \rightarrow \infty)$ was calculated according to

$$
R_{\infty}=\lim _{F \rightarrow \infty} R=1-\frac{\alpha \theta C_{0}}{C_{\text {in }}},
$$

where $C_{0}$ and $\theta$ were calculated using experimental data and linearizing (19). The parameter $\alpha$ was determined by the linear correlation of experimental data using (3).

3.2.3. Simulation of LPR Experiments from Retention Values. LPR experiments were simulated from membrane and WSP data previously published $[20,23,31]$. These data are summarized in Table 1 .

\section{Results and Discussion}

4.1. Description of LPR Experiments from Experimental Data and Semiempirical Simulation. The effect of (6) on the behavior of experimental data can be easily evidenced (see Figure 2). Thus, data denoted to be "a" correspond to the average concentration values of permeate fractions collected in the tubes whereas data denoted as "b" correspond to effective concentration values in the permeate. This first observation suggests that the experimental data report of LPR, by washing method, should be done using the accumulated concentrations instead of partial concentrations. It is clear that curve "a" is strongly affected by the experimental design of the experiments (note that for $200 \mathrm{~mL}$ in "a" 


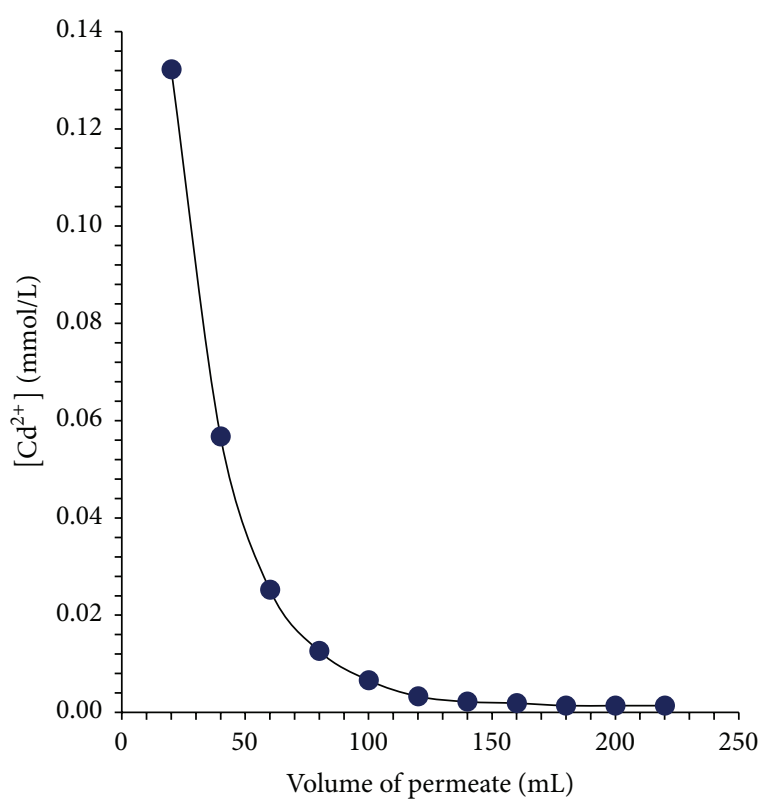

(a)

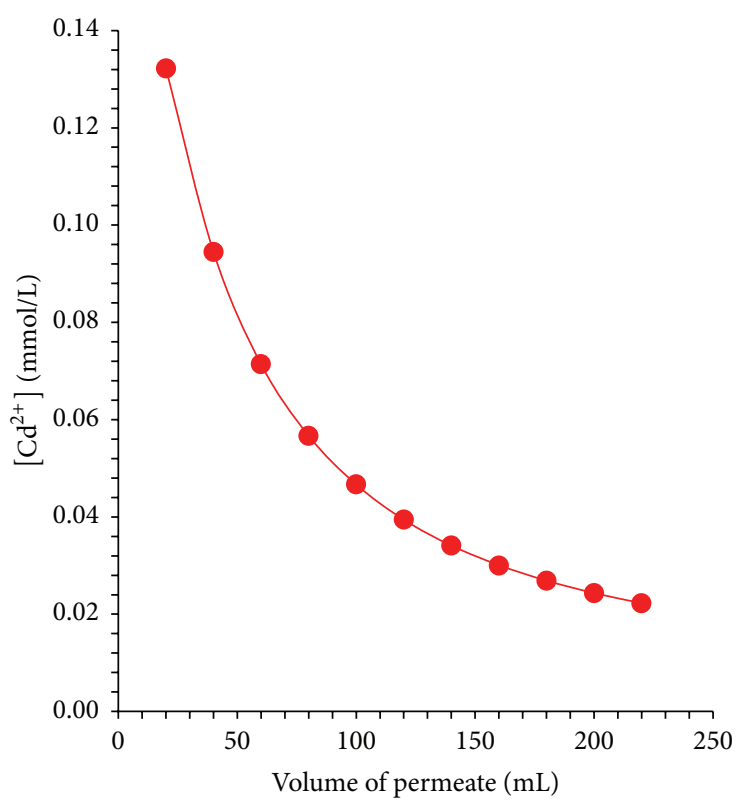

(b)

FIGURE 2: Average $\mathrm{Cd}^{2+}$ concentration values of permeate fractions collected in the tubes (a) and effective concentration values in the permeate according to (6) (b).

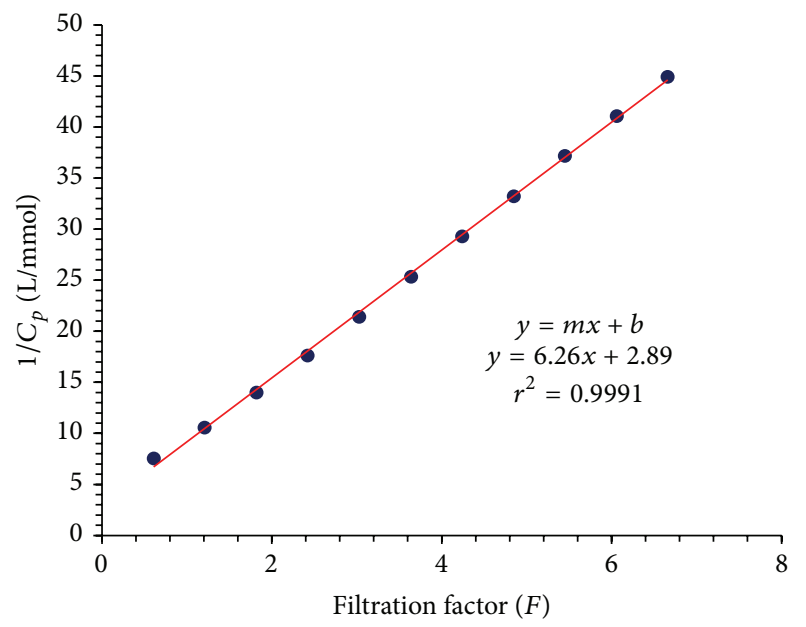

(a)

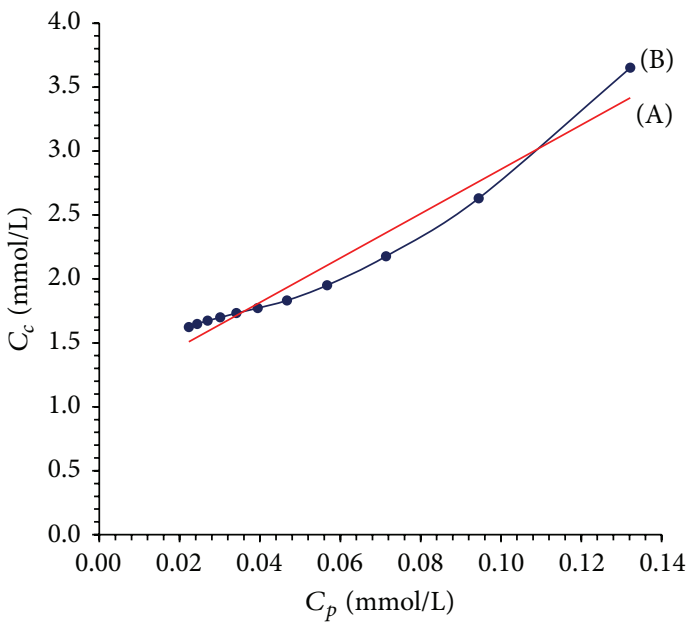

(A) Supposed behavior

(B) Experimental behavior

(b)

Figure 3: Description of experimental data by (19) (a) and evaluation of (3) from experimental data $\left(C_{c}\right.$ and $\left.C_{p}\right)$.

apparently the PII concentration in the aqueous effluent has been completely eliminated; however, from curve "b" it can be seen that PII concentration in the effluent is relatively high in comparison with initial concentration). Thus, apparently PII concentration could be identified to be zero when really this is not like that.

It can be seen that experimental data are satisfactorily described by linear form of (19) (see Figure 3(a)). However, it is clear that experimental error should be minimized in order to obtain an adequate value of $C_{0}$. On the other hand, it can be seen that the product $\alpha \theta$ can be easily determined from $b / m$ (intersection at the $y$-axis divided by slope). Though with information obtained from experimental data a simulation based on (19) can be performed, the parameter $\theta$ includes all information about interactions associated with the WSP-PII, membrane-PII, and WSP-membrane-PII interactions.

From Figure 3(b) it can be seen that (3) is not the better approximation for the description of relation between $C_{p}$ and $C_{c}$. This supposition only is valid for short ranges of $F$ and its evaluation is required depending on each system under study. 


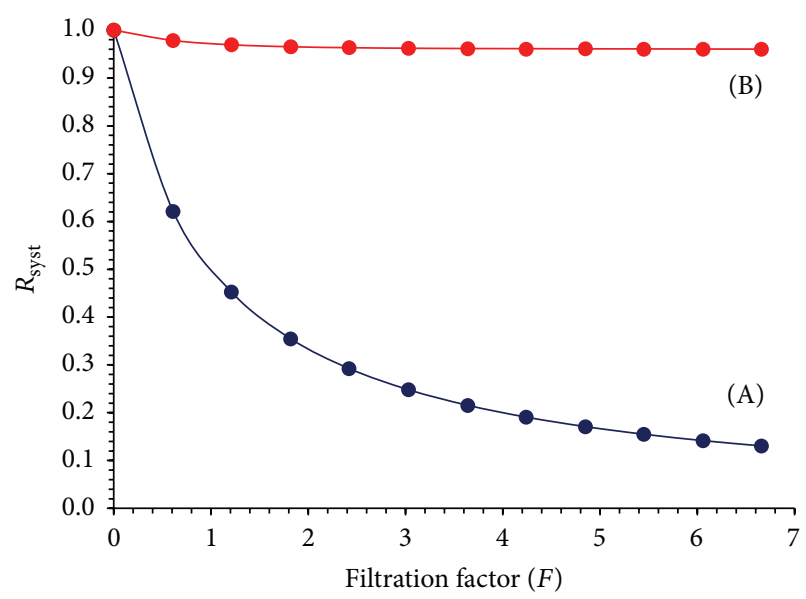

(a)

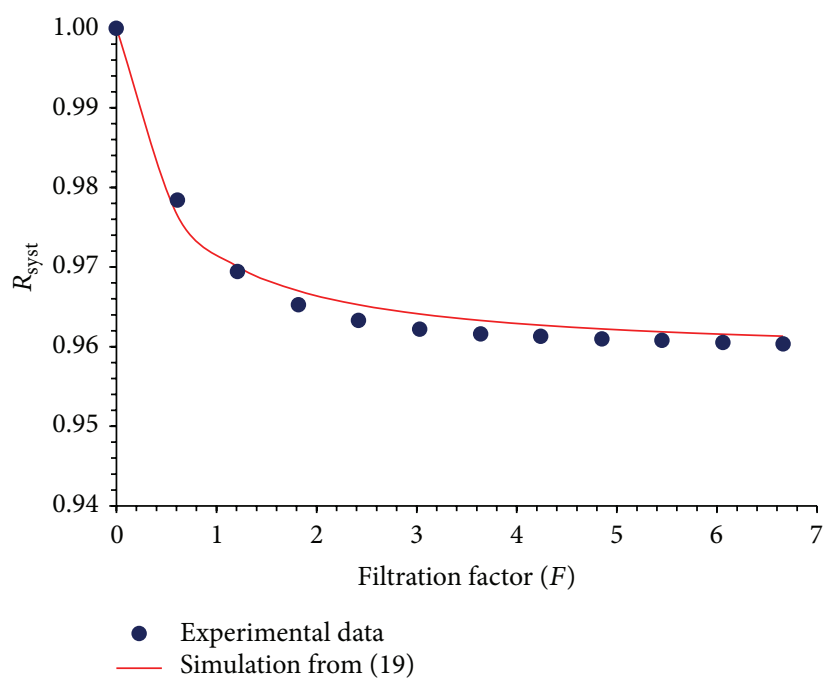

(b)

FIGURE 4: (a) Retention profiles for PSSNA-Cd and blank experiment (A and B, resp.) and (b) semiempirical modeling of retention profile from experimental data by (19).

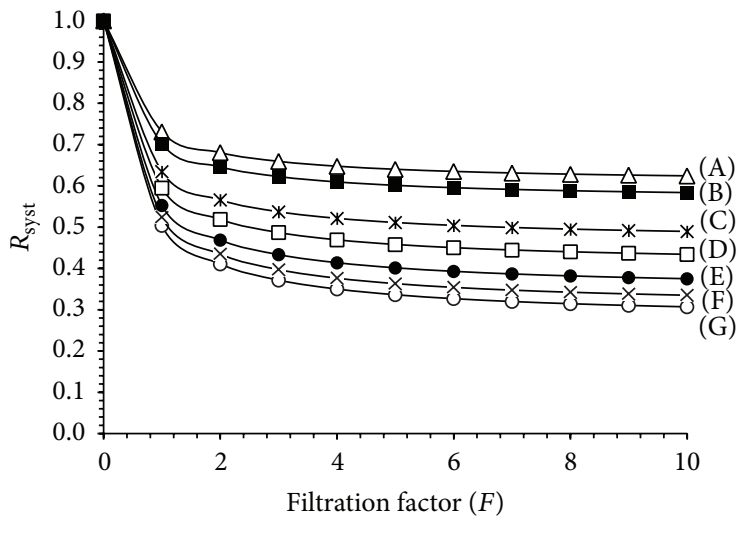

(a)

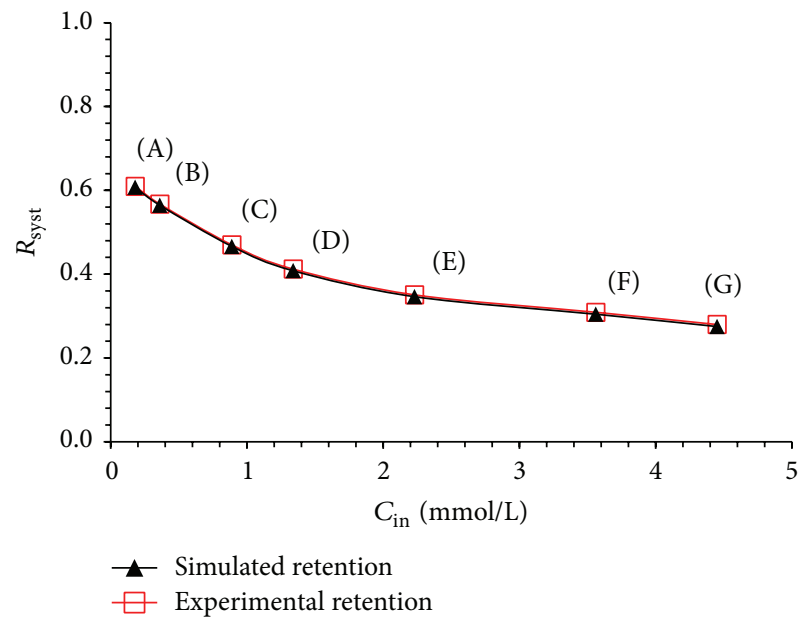

(b)

FIGURE 5: (a) Retention profiles and (b) modeling of $R_{\text {pol }}$ for PSSNA-Cd from $C_{\text {in }}$ and $R_{\text {pol }}$ data. Initial concentration values were 0.18 (A), $0.36(\mathrm{~B}), 0.89(\mathrm{C}), 1.34(\mathrm{D}), 2.23(\mathrm{E}), 3.56(\mathrm{~F})$, and $4.45(\mathrm{G})$.

In consequence, it is concluded that it is required to know an adequate value of $\theta$ and analyze the interaction parameters by new approximations.

Retention profiles for PSSNA-Cd and blank experiment are shown in Figure 4(a). In addition, from Figure 4(b) it can be seen that (19) can simulate adequately the retention profile from experimental data (semiempirical modeling). However, when retention by WSP $\left(R_{\mathrm{pol}}\right)$ at $F=6.7$ is compared with $R_{\infty}$ it is concluded that $R_{\text {pol }}<R_{\infty}\left(R_{\text {pol }}=96.5 \%\right.$ and $R_{\infty}=$ 95.9\%); thus, by modeling of LPR experiments more precise formulations of WSP necessary to remove an amount of PII can be performed when a more significant difference between $R_{\text {pol }}$ and $R_{\infty}$ is evidenced.
4.2. LPR Simulation from Retention Values. In order to simulate the retention profiles for different values of $C_{\text {in }}$ and using retention values previously published it is necessary to define some model parameters. Thus, it is clear that, by definition, $C_{0}$ can be determined from $\left(1-R_{\text {pol }}\right) C_{\text {in }}$; however, it was noted that for experiments described in the previous section this is not completely valid; therefore, a correction factor was required and this was determined by comparison of $C_{0}$ with $\left(1-R_{\text {pol }}\right) C_{\text {in }}$; thus, the correction factor calculated was 2.18 and $C_{0}$ used for each simulation was calculated to be 2.18 $\left(1-R_{\text {pol }}\right) C_{\text {in }}$.

Retention profiles are shown in Figure 5(a) and the variation of $R_{\infty}$ is shown in Figure 5(b). It can be seen 
that the model permits the description of retention profiles and the projection of new profiles from a small number of experiments and experimental data.

On the other hand, the need to use a correction factor is supported in the fact that the comparison of experimental results with theoretical predictions reveals only a partial agreement and the need to define explicitly the product $\alpha \theta$ and to refine the knowledge of changes of $\alpha$ during the elution. However, it is suggested that the differences between experiment and modeling are a result of variation in the experimental conditions which are not consistent with theoretical assumptions (i.e., conformational changes of WSP in solution, volume measures with a low precision, initial time to achieve the equilibrium pressure, polydispersity of the polymer chains, and changes of ionic strength during the elution can affect the capture of experimental data).

\section{Conclusions}

A complete modeling of LPR technique by washing method is described from the PII distribution in regions (retainer elements and passive zones) of the UF system; in consequence, semiempirical simulation of PII retention by WSP can be performed from a small amount of experimental data. In addition, it is concluded that it is required to know adequate values of $\theta$ and $\alpha$, as well as to analyze the interaction parameters by new approximations associated with molecular structure of polymer.

\section{Conflict of Interests}

The author declares that there is no conflict of interests regarding the publication of this paper.

\section{Acknowledgment}

This work was supported by internal funding of the University of Valle.

\section{References}

[1] M. Cheryan, Ultrafiltration and Microfiltration Handbook, Technomic Publishing Company, Lancaster, Pa, USA, 1998.

[2] G. Kang and Y. Cao, "Application and modification of poly(vinylidene fluoride) (PVDF) membranes-a review," Journal of Membrane Science, vol. 463, pp. 145-165, 2014.

[3] B. L. Rivas, E. D. Pereira, and I. Moreno-Villoslada, "Water-soluble polymer-metal ion interactions," Progress in Polymer Science, vol. 28, no. 2, pp. 173-208, 2003.

[4] Z. Berk, Membrane Processes: Food Process Engineering and Technology, 2nd edition, 2013.

[5] B. L. Rivas, E. D. Pereira, M. Palencia, and J. Sánchez, "Watersoluble functional polymers in conjunction with membranes to remove pollutant ions from aqueous solutions," Progress in Polymer Science, vol. 36, no. 2, pp. 294-322, 2011.

[6] K. E. Geckeler, E. Bayer, B. Y. Spivakov, V. M. Shkinev, and G. A. Vorob'eva, "Liquid-phase polymer-based retention, a new method for separation and preconcentration of elements," Analytica Chimica Acta, vol. 189, pp. 285-292, 1986.
[7] R. Molinari, T. Poerio, and P. Argurio, "Chemical and operational aspects in running the polymer assisted ultrafiltration for separation of copper(II)-citrate complexes from aqueous media," Journal of Membrane Science, vol. 295, no. 1-2, pp. 139147, 2007.

[8] E. Katsou, S. Malamis, and K. J. Haralambous, "Industrial wastewater pre-treatment for heavy metal reduction by employing a sorbent-assisted ultrafiltration system," Chemosphere, vol. 82, no. 4, pp. 557-564, 2011.

[9] R. Molinari, S. Gallo, and P. Argurio, "Metal ions removal from wastewater or washing water from contaminated soil by ultrafiltration-complexation," Water Research, vol. 38, no. 3, pp. 593-600, 2004.

[10] B. Chaufer and A. Deratani, "Removal of metal ions by complexation-ultrafiltration using water-soluble macromolecules: perspective of application to wastewater treatment," Nuclear and Chemical Waste Management, vol. 8, no. 3, pp. 175$187,1988$.

[11] Y. R. Qiu, L. J. Mao, and W. H. Wang, "Removal of manganese from waste water by complexation-ultrafiltration using copolymer of maleic acid and acrylic acid," Transactions of Nonferrous Metals Society of China (English Edition), vol. 24, no. 4, pp. 11961201, 2014.

[12] K. R. Desai and Z. V. P. Murthy, "Removal of silver from aqueous solutions by complexation-ultrafiltration using anionic polyacrylamide," Chemical Engineering Journal, vol. 185-186, pp. 187-192, 2012.

[13] J. Shao, S. Qin, J. Davidson, W. Li, Y. He, and H. S. Zhou, "Recovery of nickel from aqueous solutions by complexationultrafiltration process with sodium polyacrylate and polyethylenimine," Journal of Hazardous Materials, vol. 244-245, pp. 472477, 2013.

[14] A. Tabatabai, J. F. Scamehorn, and S. D. Christian, "Economic feasibility study of polyelectrolyte-enhanced ultrafiltration (PEUF) for water softening," Journal of Membrane Science, vol. 100, no. 3, pp. 193-207, 1995.

[15] R. Camarillo, A. Pérez, P. Cañizares, and A. de Lucas, "Removal of heavy metal ions by polymer enhanced ultrafiltration. Batch process modeling and thermodynamics of complexation reactions," Desalination, vol. 286, pp. 193-199, 2012.

[16] P. Cañizares, A. Pérez, R. Camarillo, and R. Mazarro, "Simultaneous recovery of cadmium and lead from aqueous effluents by a semi-continuous laboratory-scale polymer enhanced ultrafiltration process," Journal of Membrane Science, vol. 320, no. 1-2, pp. 520-527, 2008.

[17] J. Sabaté, M. Pujolà, and J. Llorens, "Simulation of a continuous metal separation process by polymer enhanced ultrafiltration," Journal of Membrane Science, vol. 268, no. 1, pp. 37-47, 2006.

[18] J. Llorens, M. Pujolà, and J. Sabaté, "Separation of cadmium from aqueous streams by polymer enhanced ultrafiltration: a two-phase model for complexation binding," Journal of Membrane Science, vol. 239, no. 2, pp. 173-181, 2004.

[19] S. Ahmadi, B. Batchelor, and S. S. Koseoglu, "Removal of toxic heavy metal ions from metal finishing industry effluents by micellar enhanced ultrafiltration technology," Journal of Hazardous Materials, vol. 28, no. 1-2, p. 225, 1991.

[20] M. Palencia, B. L. Rivas, E. Pereira, A. Hernández, and P. Prádanos, "Study of polymer-metal ion-membrane interactions in liquid-phase polymer-based retention (LPR) by continuous diafiltration," Journal of Membrane Science, vol. 336, no. 1-2, pp. 128-139, 2009. 
[21] K. E. Geckeler, "Polymer-metal complexes for environmental protection. Chemoremediation in the aqueous homogeneous phase," Pure and Applied Chemistry, vol. 73, no. 1, pp. 129-136, 2001.

[22] K. E. Geckeler, V. M. Shkinev, and B. Y. Spivakov, "Liquid-phase polymer-based retention (LPR) - a new method for selective ion separation," Separation and purification methods, vol. 17, no. 2, pp. 105-140, 1988.

[23] Y. Uludag, H. Ö. Özbelge, and L. Yilmaz, "Removal of mercury from aqueous solutions via polymer-enhanced ultrafiltration," Journal of Membrane Science, vol. 129, no. 1, pp. 93-99, 1997.

[24] V. M. Shkinev, V. N. Gomolitskii, B. Y. Spivakov, K. E. Geckeler, and E. Bayer, "Determination of trace heavy metals in waters by atomic-absorption spectrometry after preconcentration by liquid-phase polymer-based retention," Talanta, vol. 36, no. 8 , pp. 861-863, 1989.

[25] S. Ahmadi, B. Batchelor, and S. S. Koseoglu, "The diafiltration method for the study of the binding of macromolecules to heavy metals," Journal of Membrane Science, vol. 89, no. 3, pp. 257-265, 1994.

[26] S. D. Alexandratos and X. Zhu, "High-affinity ion-complexing polymer-supported reagents: immobilized phosphate ligands and their affinity for the uranyl ion," Reactive and Functional Polymers, vol. 67, no. 5, pp. 375-382, 2007.

[27] W. M. Anspach and J. A. Marinsky, "Complexing of nickel(II) and cobalt(II) by a polymethacrylic acid gel and its linear polyelectrolyte analog," Journal of Physical Chemistry, vol. 79, no. 5, pp. 433-439, 1975.

[28] K. H. Choo, S. C. Han, S. J. Choi et al., "Use of chelating polymers to enhance manganese removal in ultrafiltration for drinking water treatment," Journal of Industrial and Engineering Chemistry, vol. 13, no. 2, pp. 163-169, 2007.

[29] D. Zamariotto, B. Lakard, P. Fievet, and N. Fatin-Rouge, "Retention of $\mathrm{Cu}(\mathrm{II})$ - and $\mathrm{Ni}(\mathrm{II})$ - polyaminocarboxylate complexes by ultrafiltration assisted with polyamines," Desalination, vol. 258, no. 1-3, pp. 87-92, 2010.

[30] L. Dambies, A. Jaworska, G. Zakrzewska-Trznadel, and B. Sartowska, "Comparison of acidic polymers for the removal of cobalt from water solutions by polymer assisted ultrafiltration," Journal of Hazardous Materials, vol. 178, no. 1-3, pp. 988-993, 2010.

[31] M. Palencia, B. L. Rivas, and E. Pereira, "Polymer-enhanced ultrafiltration: counterion distribution and its relation with the divalent metal-ion retention properties by sulfonic acid polyelectrolytes," Polymer Bulletin, vol. 67, no. 7, pp. 1123-1138, 2011.

[32] M. Vera, M. Palencia, and E. Enrique Combatt, "Estudio de la capacidad de retención de boro disponible en suelos mediante membranas funcionales con cadenas de polioles," Revista Temas Agrarios, vol. 19, no. 1, pp. 98-107, 2014.

[33] A. Deshkovski, S. Obukhov, and M. Rubinstein, "Counterion phase transitions in dilute polyelectrolyte solutions," Physical Review Letters, vol. 86, no. 11, pp. 2341-2344, 2001.

[34] A. V. Dobrynin, "Effect of counterion condensation on rigidity of semiflexible polyelectrolytes," Macromolecules, vol. 39, no. 26, pp. 9519-9527, 2006.

[35] G. S. Manning, "Counterion binding in polyelectrolyte theory," Accounts of Chemical Research, vol. 12, no. 12, pp. 443-449, 1979.

[36] G. S. Manning, "Limiting laws and counterion condensation in polyelectrolyte solutions. 7. Electrophoretic mobility and conductance," Journal of Physical Chemistry, vol. 85, no. 11, pp. 15061515, 1981. 

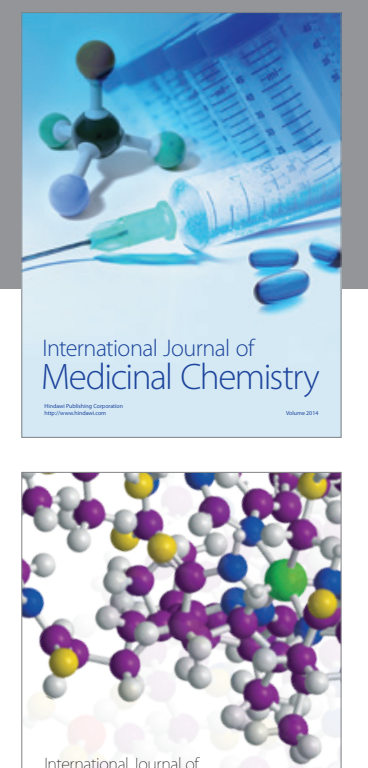

\section{Carbohydrate} Chemistry

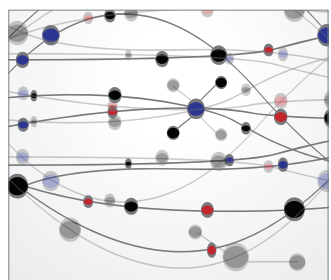

The Scientific World Journal
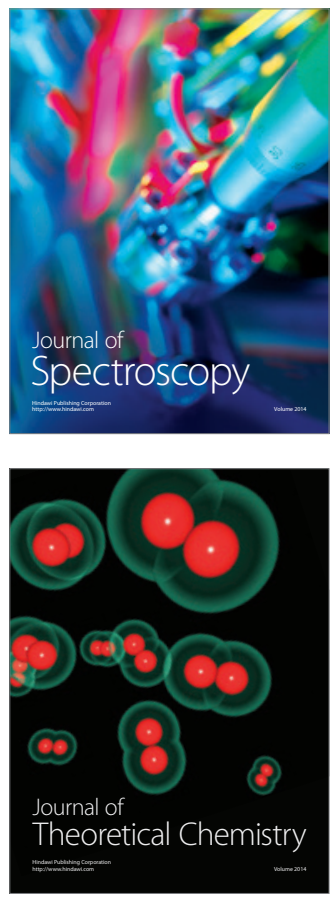
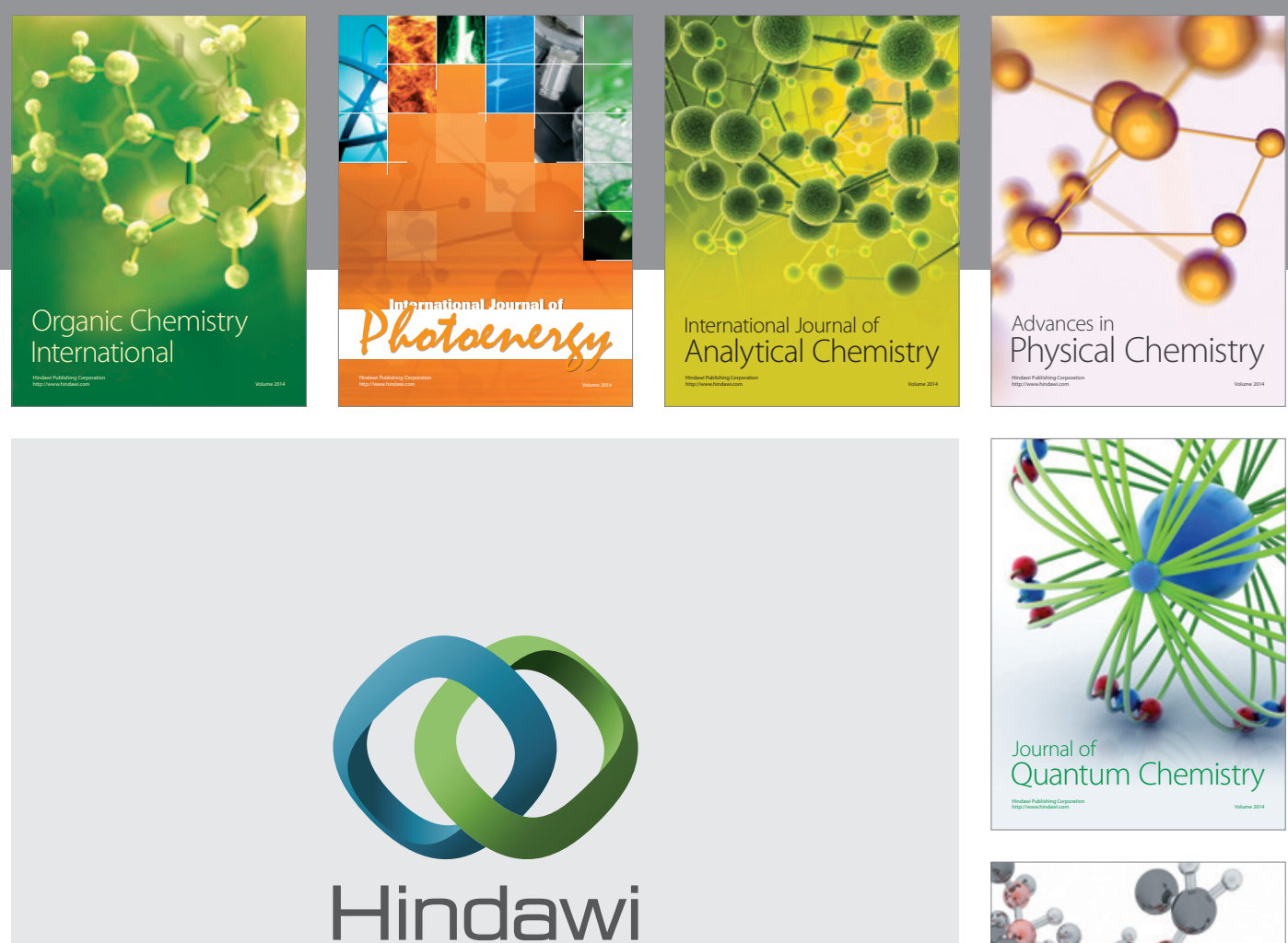

Submit your manuscripts at

http://www.hindawi.com

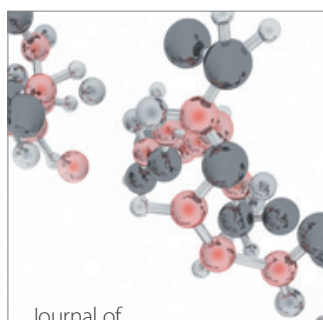

Analytical Methods

in Chemistry

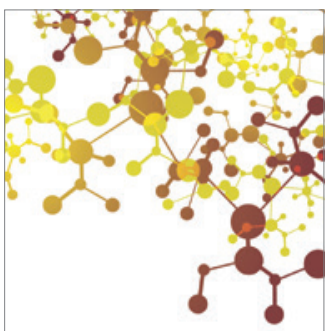

Journal of

Applied Chemistry

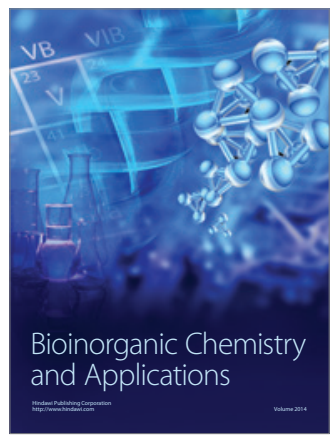

Inorganic Chemistry
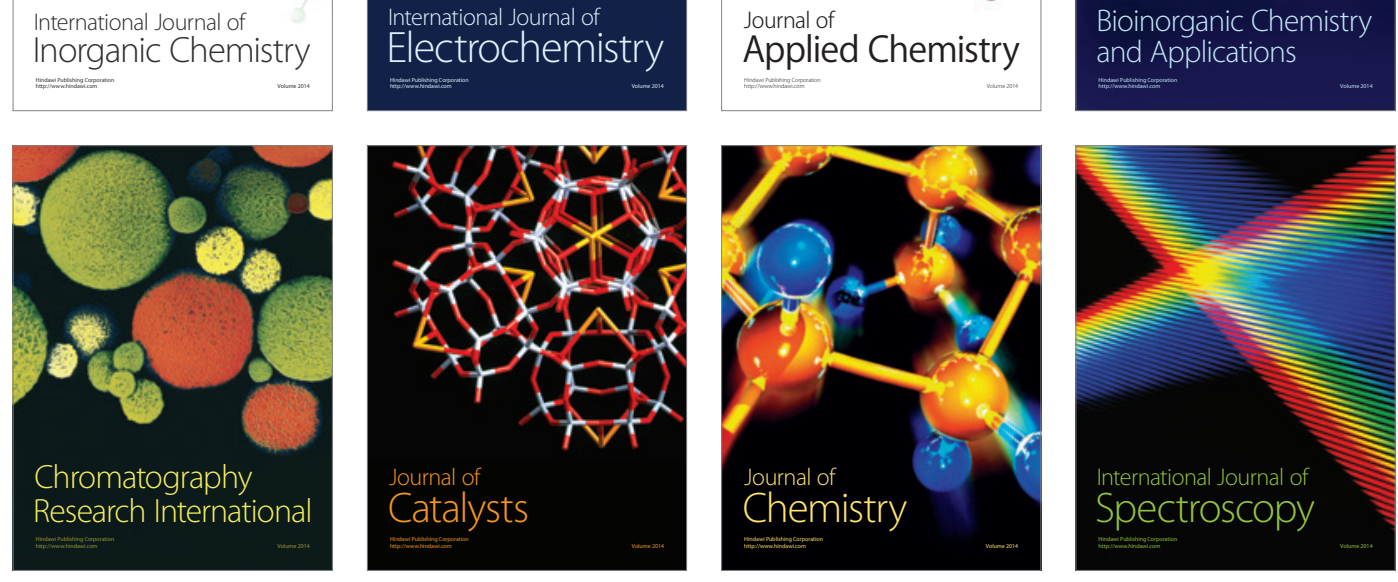